Homology, Homotopy and Applications, vol. 17(2), 2015, pp.1-12

\title{
ON THE MIDDLE DIMENSIONAL HOMOLOGY CLASSES OF EQUILATERAL POLYGON SPACES
}

\author{
YASUHIKO KAMIYAMA \\ (communicated by R. James Milgram)
}

\begin{abstract}
Let $M_{n}$ be the configuration space of equilateral polygonal linkages with $n$ vertices in the Euclidean plane $\mathbb{R}^{2}$. We consider the case where $n$ is odd and set $n=2 m+1$.

In spite of the long history of research, the homology classes in $H_{m-1}\left(M_{n} ; \mathbb{Z}\right)$ are mysterious and not well-understood. Let $\tau: M_{n} \rightarrow M_{n}$ be the involution induced by complex conjugation. In this paper, we determine the representation matrix of the homomorphism $\tau_{*}: H_{m-1}\left(M_{n} ; \mathbb{Z}\right) \rightarrow H_{m-1}\left(M_{n} ; \mathbb{Z}\right)$ with respect to a basis of $H_{m-1}\left(M_{n} ; \mathbb{Z}\right)$.
\end{abstract}

\section{Introduction}

We consider the configuration space $M_{n}$ of equilateral polygonal linkages with $n(n \geqslant 3)$ vertices, each edge having length 1 in the Euclidean plane $\mathbb{R}^{2}$ modulo the orientation-preserving isometry group. We remark that $M_{n}$ has the following description:

$$
M_{n}=\left\{\begin{array}{l|l}
\left(z_{1}, \ldots, z_{n-1}\right) \in\left(S^{1}\right)^{n-1} \mid \sum_{i=1}^{n-1} z_{i}=1
\end{array}\right\} .
$$

Here $z_{i} \in S^{1} \subset \mathbb{C}$ denote the unit vectors in the direction of the sides of a polygon.

More generally, starting in $[\mathbf{5}, \mathbf{9}, \mathbf{1 5}]$, the topology of the configuration space of polygons of arbitrary edge lengths has been considered by many authors. For example, the homology groups were determined in [4]. The study culminated in the proof by $[\mathbf{3}]$ and $[\mathbf{1 3}]$ of a conjecture by Kevin Walker which states that one can recover relative lengths of edges from the integral cohomology ring of the configuration space.

While they made clever arguments to distinguish the cohomology rings, the explicit structure of the rings is not determined completely. For example, consider $M_{n}$ for odd $n=2 m+1$. In this case, $M_{n}$ is a connected closed manifold of dimension $n-3$. The

Received April 20, 2015, revised March 27, 2015; published on July 9, 2015.

2010 Mathematics Subject Classification: 58D29, 55R80.

Key words and phrases: polygon space, involution, homology class.

Article available at http://dx.doi.org/10.4310/HHA.2015.v17.n2.a1

Copyright (C) 2015, International Press. Permission to copy for private use granted. 
homology groups $H_{*}\left(M_{n} ; \mathbb{Z}\right)$ are torsion free and the Poincaré polynomial is given by

$$
P S\left(M_{n}\right)=\sum_{q=0}^{m-2}\left(\begin{array}{c}
n-1 \\
q
\end{array}\right) t^{q}+2\left(\begin{array}{c}
n-1 \\
m-1
\end{array}\right) t^{m-1}+\sum_{q=m}^{n-3}\left(\begin{array}{c}
n-1 \\
q+2
\end{array}\right) t^{q} .
$$

Moreover, for the natural inclusion

$$
i: M_{n} \rightarrow\left(S^{1}\right)^{n-1},
$$

the induced homomorphism

$$
i_{*}: H_{q}\left(M_{n} ; \mathbb{Z}\right) \rightarrow H_{q}\left(\left(S^{1}\right)^{n-1} ; \mathbb{Z}\right)
$$

is an isomorphism for $q \leqslant m-2$ and an epimorphism for $q=m-1$. (See $[\mathbf{4}, \mathbf{8}]$.) The following number plays a central role in this paper:

$$
D_{n}=\left(\begin{array}{c}
n-1 \\
m-1
\end{array}\right)
$$

We have $\operatorname{rank} H_{m-1}\left(\left(S^{1}\right)^{n-1} ; \mathbb{Z}\right)=D_{n}$ but rank $H_{m-1}\left(M_{n} ; \mathbb{Z}\right)=2 D_{n}$. This implies that $H_{m-1}\left(M_{n} ; \mathbb{Z}\right)$ contains homology classes which do not come from $\left(S^{1}\right)^{n-1}$. (See also (13) in $§ 2$.) The classes cause difficulty in determining the ring $H^{*}\left(M_{n} ; \mathbb{Z}\right)$.

To consider the homology classes, recall that $M_{n}$ comes with a natural involution

$$
\tau: M_{n} \rightarrow M_{n}, \quad \tau\left(z_{1}, \ldots, z_{n-1}\right)=\left(\bar{z}_{1}, \ldots, \bar{z}_{n-1}\right),
$$

induced by complex conjugation. It is sometimes guessed that there might be a basis

$$
\left\langle u_{1}, \ldots, u_{D_{n}}, v_{1}, \ldots, v_{D_{n}}\right\rangle
$$

of $H_{m-1}\left(M_{n} ; \mathbb{Z}\right)$ such that $\tau_{*}\left(u_{i}\right)=v_{i}$ for all $1 \leqslant i \leqslant D_{n}$. In other words, we fix a basis of $H_{m-1}\left(M_{n} ; \mathbb{Z}\right)$ and denote by $A$ the representation matrix of the homomorphism

$$
\tau_{*}: H_{m-1}\left(M_{n} ; \mathbb{Z}\right) \rightarrow H_{m-1}\left(M_{n} ; \mathbb{Z}\right)
$$

with respect to the basis. Then it is guessed that there might exist $S \in G L(n, \mathbb{Z})$ such that

$$
S^{-1} A S=\underset{D_{n}}{\oplus}\left(\begin{array}{ll}
0 & 1 \\
1 & 0
\end{array}\right)
$$

Now we pose the following:

Question 1. Is (6) true for odd $n$ ?

The purpose of this paper is to give a negative answer to Question 1. This paper is organized as follows. In $\S 2$ we state our main results (Theorem 2 and Corollary 3 ). Theorem 2 is essentially equivalent to Theorem 5 . Theorem 6 is a key result for Theorem 5 and Proposition 8 is a key result for Theorem 6 . In $\S 3$ we prove Proposition 8 and in $\S 4$ we prove Theorem 6 . In $\S 5$ we prove Theorem 5 and in $\S 6$ we prove our main results.

\section{Statements of the main results}

Hereafter we assume that $n$ is an odd number which satisfies $n \geqslant 5$. We set $n=$ $2 m+1$. 
Theorem 2. We fix a basis of $H_{m-1}\left(M_{n} ; \mathbb{Z}\right)$ and denote by $A$ the representation matrix of the homomorphism (5) with respect to the basis. We set

$$
\alpha_{n}=\sum_{i=0}^{m-1} 2^{m-1-i}\left(\begin{array}{c}
2 i \\
i
\end{array}\right) \quad \text { and } \quad \beta_{n}=D_{n}-\alpha_{n},
$$

where $D_{n}$ is defined in (3). Then there exists $S \in G L(n, \mathbb{Z})$ such that

$$
S^{-1} A S=\underset{\alpha_{n}}{\oplus}\left(\begin{array}{ll}
0 & 1 \\
1 & 0
\end{array}\right) \oplus \underset{\beta_{n}}{\oplus}\left(\begin{array}{cc}
1 & 0 \\
0 & -1
\end{array}\right) .
$$

Corollary 3. Question 1 is false for odd $n$ greater than or equal to 7.

Remark 4. (i) It is reasonable that Question 1 is true for $n=5$. In fact, $M_{5}$ is homeomorphic to a connected closed orientable surface of genus 4 . We number the four handles appropriately and denote them by $h_{i}$ for $1 \leqslant i \leqslant 4$. Then $\tau$ acts on $M_{5}$ by exchanging $h_{1}$ with $h_{3}$ and $h_{2}$ with $h_{4}$. (See, for example, [9].)

(ii) It is known (see, for example, [14, A082590]) that $\alpha_{n}$ satisfies the following equation:

$$
\frac{1}{(1-2 x) \sqrt{1-4 x}}=\sum_{i=0}^{\infty} \alpha_{2 i+3} x^{i} .
$$

Recall that an integral square matrix $P$ with $P^{2}=I$ has a normal form. (See Lemma 11 in $\S 6$.) Thanks to this, we can restate Theorem 2 as the following:

Theorem 5. An elementary divisor of the homomorphism

$$
1-\tau_{*}: H_{m-1}\left(M_{n} ; \mathbb{Z}\right) \rightarrow H_{m-1}\left(M_{n} ; \mathbb{Z}\right)
$$

is 0,1 , or 2 . Moreover, the numbers of the elementary divisors are given by the following table.

\begin{tabular}{|c|c|c|c|}
\hline elementary divisor & 0 & 1 & 2 \\
\hline number & $D_{n}$ & $\alpha_{n}$ & $\beta_{n}$ \\
\hline
\end{tabular}

Table 1: The numbers of the elementary divisors of $1-\tau_{*}$

The proof of Theorem 5 consists of three steps. We set

$$
\bar{M}_{n}=M_{n} / \tau \text {. }
$$

Moreover, let $\tau$ act on $S^{1}$ antipodally, and set

$$
E_{n}=M_{n} \times_{\tau} S^{1} .
$$

The homology groups $H_{*}\left(\bar{M}_{n} ; \mathbb{Z}\right)$ are determined in [7] and the cohomology ring $H^{*}\left(\bar{M}_{n} ; \mathbb{Z}_{2}\right)$ is determined in $[\mathbf{6}]$ (see also Theorem 7 below).

(i) Using the Gysin sequence of the bundle

$$
S^{1} \rightarrow E_{n} \rightarrow \bar{M}_{n},
$$

we determine $H_{*}\left(E_{n} ; \mathbb{Z}_{2}\right)$. (See $(20)$ ). Here we recall that when $n$ is odd, $\tau$ is a fixed-point-free involution. Hence (9) is an $S^{1}$-bundle. 
(ii) With some more computations, we determine $H_{*}\left(E_{n} ; \mathbb{Z}\right)$. (See Theorem 6 ).

(iii) Using the Wang sequence of the bundle

$$
M_{n} \rightarrow E_{n} \rightarrow S^{1}
$$

we determine elementary divisors of the homomorphism (8). (See $\S 5$ ).

The following theorem is a key to proving Theorem 5 .

Theorem 6. (i) Let $x \in H_{*}\left(E_{n} ; \mathbb{Z}\right)$ be a torsion element. Then we have $2 x=0$. Therefore, for each $q$, the module $H_{q}\left(E_{n} ; \mathbb{Z}\right)$ has the form

$$
\underset{a_{q}}{\oplus} \mathbb{Z} \oplus \underset{b_{q}}{\oplus} \mathbb{Z}_{2}
$$

for some $a_{q}, b_{q} \in \mathbb{N} \cup\{0\}$.

(ii) We set

$$
\phi_{n}=\sum_{\substack{q=0 \\
q \text { even }}}^{m-2}\left(\begin{array}{c}
n-1 \\
q
\end{array}\right) t^{q}+D_{n} t^{m-1}+\sum_{\substack{q=m \\
q \text { odd }}}^{n-4}\left(\begin{array}{c}
n-1 \\
q+2
\end{array}\right) t^{q} .
$$

Then we have

$$
P S_{\mathbb{Q}}\left(E_{n}\right)=(1+t) \phi_{n}
$$

Note that this determines $a_{q}$ in (i).

(iii) We set

$$
\Gamma\left(E_{n}\right)=\sum_{q=0}^{\infty} b_{q} t^{q}
$$

where $b_{q}$ is defined in (i). Then we have

$$
\Gamma\left(E_{n}\right)=\sum_{\substack{q=0 \\
q \text { odd }}}^{m-2}\left(\begin{array}{c}
n-1 \\
q
\end{array}\right) t^{q}+\beta_{n} t^{m-1}+\sum_{\substack{q=m \\
q \text { even }}}^{n-3}\left(\begin{array}{c}
n-1 \\
q+2
\end{array}\right) t^{q}
$$

where $\beta_{n}$ is defined in Theorem 2.

We prove Theorem 6 in $\S 4$. We can prove (i) and (ii) of the theorem by standard arguments. But for (iii), we need to prove Proposition 8 below. To state the proposition, we recall the following:

Theorem 7 ([6, Cor. 9.2 and Prop. 9.3]). (i) The mod 2 cohomology ring of $\bar{M}_{n}$ is

$$
H^{*}\left(\bar{M}_{n} ; \mathbb{Z}_{2}\right)=\mathbb{Z}_{2}\left[R_{n}, V_{1}, \ldots, V_{n-1}\right] / \mathcal{J}_{n},
$$

where $R_{n}$ and $V_{1}, \ldots, V_{n-1}$ are of degree 1 and $\mathcal{J}_{n}$ is the ideal generated by the three families

(R1) $V_{i}^{2}+R_{n} V_{i} \quad$ where $i=1, \ldots, n-1$,

(R2) $\prod_{i \in S} V_{i} \quad$ where $S \subset\{1, \ldots, n-1\}$ is such that $m \leqslant|S|$, 
(R3) $\sum_{S \subset L} R_{n}^{|L-S|-1} \prod_{i \in S} V_{i} \quad$ where $L \subset\{1, \ldots, n-1\}$ is such that $m+1 \leqslant|L|$.

The symbol $S$ in (R3) runs over all subsets of $L$ including the empty set. By (R2) a term of the sum in (R3) vanishes if $m \leqslant|S|$.

(ii) Let $\xi \rightarrow \bar{M}_{n}$ be the line bundle associated with the regular 2 -cover $\pi: M_{n} \rightarrow \bar{M}_{n}$. Then we have $R_{n}=w_{1}(\xi)$.

For a field $K$ and a space $X$, let $P S_{K}(X)$ be the Poincaré polynomial of $X$ with coefficients in $K$. As a corollary of Theorem 7 (i), we have

$$
P S_{\mathbb{Z}_{2}}\left(\bar{M}_{n}\right)=\sum_{q=0}^{m-1}\left(\sum_{i=0}^{q}\left(\begin{array}{c}
n-1 \\
i
\end{array}\right)\right) t^{q}+\sum_{q=m}^{n-3}\left(\sum_{i=0}^{n-3-q}\left(\begin{array}{c}
n-1 \\
i
\end{array}\right)\right) t^{q} .
$$

Proposition 8. We define

$$
\lambda_{q}=\operatorname{rank}\left[\cup R_{n}^{2}: H^{q}\left(\bar{M}_{n} ; \mathbb{Z}_{2}\right) \rightarrow H^{q+2}\left(\bar{M}_{n} ; \mathbb{Z}_{2}\right)\right] .
$$

We set

$$
\gamma_{n}=\sum_{i=0}^{m-2}\left(\begin{array}{c}
n-1 \\
i
\end{array}\right)
$$

Then the following result holds.

$$
\lambda_{q}= \begin{cases}\operatorname{dim} H^{q}\left(\bar{M}_{n} ; \mathbb{Z}_{2}\right), & 0 \leqslant q \leqslant m-3, \\ \gamma_{n}-\beta_{n}, & q=m-2, \\ \operatorname{dim} H^{q+2}\left(\bar{M}_{n} ; \mathbb{Z}_{2}\right), & m-1 \leqslant q \leqslant 2 m-4 .\end{cases}
$$

The proposition implies that the homomorphism

$$
\cup R_{n}^{2}: H^{q}\left(\bar{M}_{n} ; \mathbb{Z}_{2}\right) \rightarrow H^{q+2}\left(\bar{M}_{n} ; \mathbb{Z}_{2}\right)
$$

is injective for $0 \leqslant q \leqslant m-3$ and surjective for $m-1 \leqslant q \leqslant 2 m-4$. The only difficult case is that $\cup R_{n}^{2}$ jumps over $H^{m-1}\left(\bar{M}_{n} ; \mathbb{Z}_{2}\right)$. Note that $m-1=\frac{1}{2} \operatorname{dim} \bar{M}_{n}$.

Additionally, we will reduce Proposition 8 to Lemma 10 in $\S 3$, which is a problem in linear algebra and combinatorics.

Before we leave this section, we give the following:

Remark 9. Note that (2) realizes $M_{n}$ as a codimension-two submanifold of $\left(S^{1}\right)^{n-1}$. On the other hand, it is also possible to realize $M_{n}$ as a codimension-one submanifold of $\left(S^{1}\right)^{n-2}$. More precisely, there exists a compact $(n-2)$-dimensional submanifold $W_{n}$ of $\left(S^{1}\right)^{n-2}$ with boundary such that $\partial W_{n}=M_{n}$. (See [11] for more details.) Let $X^{(m-1)}$ be the $(m-1)$-skeleton of $X:=\left(S^{1}\right)^{n-1}$, where we give a cell structure of $X$ by the product complex of $S^{1}=e^{0} \cup e^{1}$. Then it is proved in [11] that there is a homotopy equivalence $W_{n} \simeq X^{(m-1)}$. Consider the homology long exact sequence of 
the pair $\left(W_{n}, M_{n}\right)$ :

$$
\begin{aligned}
& \longrightarrow H_{m}\left(W_{n} ; \mathbb{Z}\right) \longrightarrow H_{m}\left(W_{n}, M_{n} ; \mathbb{Z}\right) \longrightarrow H_{m-1}\left(M_{n} ; \mathbb{Z}\right) \longrightarrow H_{m-1}\left(W_{n} ; \mathbb{Z}\right) \longrightarrow 0 \\
& \mathbb{I R} \quad \mathbb{R} \quad \mathbb{R} \\
& \begin{array}{ccc}
H_{m}\left(X^{(m-1)} ; \mathbb{Z}\right) & H^{m-1}\left(W_{n} ; \mathbb{Z}\right) & \underset{D_{n}}{\oplus} \mathbb{Z} \\
\| & \mathbb{R}
\end{array} \\
& 0 \quad \underset{D_{n}}{\oplus} \mathbb{Z}
\end{aligned}
$$

Here we used Lefschetz duality.

The involution (4) naturally extends to an involution

$$
\tau: W_{n} \rightarrow W_{n} .
$$

In contrast to (4), the involution (14) has fixed points. In fact, the fixed point set coincides with the set of critical points of the restriction $f \mid W_{n}-M_{n}$, which is a Morse function, of a certain function $f: W_{n} \rightarrow \mathbb{R}$. (See $[\mathbf{1 1}]$ for more details.)

Now we remark that (7) gives information on the direct summands of (13). We also have a question: How does (7) reflect the information on the fixed point set of (14)? The author is grateful to the referee for suggesting these interesting comments.

\section{Proof of Proposition 8}

(i) The case for $0 \leqslant q \leqslant m-3$. Among the relations in Theorem 7 , only (R1) is effective for $H^{q+2}\left(\bar{M}_{n} ; \mathbb{Z}_{2}\right)$. Hence (12) is injective.

(ii) The case for $m-1 \leqslant q \leqslant 2 m-4$. (R2) tells us that any element $x \in H^{q+2}\left(\bar{M}_{n} ; \mathbb{Z}_{2}\right)$ is of the form $x=R_{n}^{q-m+3} y$ for some $y \in \mathbb{Z}_{2}\left[R_{n}, V_{1}, \ldots, V_{n-1}\right]$. Since $q-m+3 \geqslant 2$, (12) is surjective.

For the rest of this section, we consider the case $q=m-2$. For that purpose, we need to study the ideal $\mathcal{J}_{n}$ more carefully. We number $(m+1)$-element subsets of $\{1, \cdots, n-1\}$ as $L_{1}, \cdots, L_{D_{n}}$, where we understand $D_{n}$ in $(3)$ as $\left(\begin{array}{c}n-1 \\ m+1\end{array}\right)$. For each $L_{i}$, we consider Theorem 7 (R3) and write the relation as

$$
p_{i}=f_{i}
$$

where $p_{i}$ is the sum of monomials defined by $(m-1)$-element subsets of $L_{i}$ and $f_{i}$ is the sum of the monomials defined by $k$-element subsets of $L_{i}$ for all $k \leqslant m-2$. We set

$$
X_{m+1}=\left\{\xi=\left(\xi_{1}, \cdots, \xi_{D_{n}}\right) \in\left(\mathbb{Z}_{2}\right)^{D_{n}} \mid \sum_{i=1}^{D_{n}} \xi_{i} p_{i}=0 \text { in } \mathbb{Z}_{2}\left[R_{n}, V_{1}, \ldots, V_{n-1}\right]\right\} .
$$

Lemma 10. (i) We have $\operatorname{dim} X_{m+1}=\beta_{n}$.

(ii) We fix a basis $\Omega_{m+1}$ of $X_{m+1}$. For $\xi \in \Omega_{m+1}$, we set

$$
F_{\xi}=\sum_{i=1}^{D_{n}} \xi_{i} f_{i}
$$

Then the elements of the set $\left\{F_{\xi} \mid \xi \in \Omega_{m+1}\right\}$ are linearly independent in $\mathbb{Z}_{2}\left[R_{n}, V_{1}, \ldots, V_{n-1}\right]$. 
Proof. (i) Consider the $\mathbb{Z}_{2}$-vector space $C_{k}$ with $k$-element subsets of $\{1, \cdots, n-1\}$ as a basis. Let $\partial_{k}: C_{k} \rightarrow C_{k-2}$ be the linear map defined on the basis by the formula

$$
\partial_{k}(\Delta):=\Gamma_{1}+\cdots+\Gamma_{\left(\begin{array}{c}
k \\
k-2
\end{array}\right)},
$$

where $\Gamma_{i}$ are the $(k-2)$-element subsets of $\Delta$. We regard $\left\langle L_{1}, \ldots, L_{D_{n}}\right\rangle$ as a basis of $C_{m+1}$. Forgetting $R_{n}$, we regard $p_{i}$ as an element of $C_{m-1}$. From the definition of $p_{i}$, we have $\partial_{m+1}\left(L_{i}\right)=p_{i}$. Thus there is an isomorphism $X_{m+1} \cong \operatorname{Ker} \partial_{m+1}$.

Consider the chain complex

$$
\cdots \stackrel{\partial_{m+3}}{\longrightarrow} C_{m+1} \stackrel{\partial_{m+1}}{\longrightarrow} C_{m-1} \stackrel{\partial_{m-1}}{\longrightarrow} \cdots \stackrel{\partial_{\varepsilon+2}}{\longrightarrow} C_{\varepsilon} \longrightarrow 0,
$$

where $\varepsilon$ is 0 or 1 according to whether $m$ is odd or even. Let $H_{q}$ be the $q$ th homology group of (15). Then [1, Theorem 3.2] tells us that $H_{\varepsilon+2 i}=0$ for all $i \in \mathbb{N} \cup\{0\}$. In fact, the middle term in the sense of $[\mathbf{1}, \mathrm{p} .557]$ is $C_{m}$ in the other chain complex

$$
\cdots \stackrel{\partial_{m+2}}{\longrightarrow} C_{m} \stackrel{\partial_{m}}{\longrightarrow} C_{m-2} \stackrel{\partial_{m-2}}{\longrightarrow} \cdots \stackrel{\partial_{\delta+2}}{\longrightarrow} C_{\delta} \longrightarrow 0
$$

and this is the only term which gives $H_{q} \neq 0$.

We construct the following long exact sequence from (15):

$0 \longrightarrow$ Ker $\partial_{m+1} \longrightarrow C_{m+1} \stackrel{\partial_{m+1}}{\longrightarrow} C_{m-1} \stackrel{\partial_{m-1}}{\longrightarrow} \cdots \stackrel{\partial_{\varepsilon+2}}{\longrightarrow} C_{\varepsilon} \longrightarrow 0$.

Computing the Euler characteristic of this, we have

$$
\operatorname{dim} \operatorname{Ker} \partial_{m+1}=D_{n}-d_{n},
$$

where we set

$$
d_{n}=\sum_{i=0}^{\lfloor(m-1) / 2\rfloor}(-1)^{i}\left(\begin{array}{c}
2 m \\
m-1-2 i
\end{array}\right)
$$

for $n=2 m+1$. It is easy to see (see also [14, A082590]) that

$$
\sum_{k=0}^{m}\left(\begin{array}{c}
2 m \\
k
\end{array}\right) \sin \frac{m-k}{2} \pi=\sum_{k=0}^{m-1} 2^{m-1-k}\left(\begin{array}{c}
2 k \\
k
\end{array}\right) .
$$

We have from this that $d_{n}=\alpha_{n}$ for all odd numbers $n$. Thus we complete the proof of Lemma 10 (i).

(ii) Writing $\xi \in \Omega_{m+1}$ in the column vector, we define a $D_{n} \times \beta_{n}$ matrix $P$. We have

$$
\left(\begin{array}{lll}
p_{1} & \cdots & p_{D_{n}}
\end{array}\right) P=\left(\begin{array}{lll}
0 & \cdots & 0
\end{array}\right)
$$

Assume that

$$
\left(\begin{array}{lll}
f_{1} & \cdots & f_{D_{n}}
\end{array}\right) P\left(\begin{array}{c}
x_{1} \\
\vdots \\
x_{\beta_{n}}
\end{array}\right)=0 .
$$

We must show that $x_{1}=\cdots=x_{\beta_{n}}=0$. Using (16) and (17), we can write

$$
\left(\begin{array}{lll}
p_{1}+f_{1} & \cdots & p_{D_{n}}+f_{D_{n}}
\end{array}\right) P\left(\begin{array}{c}
x_{1} \\
\vdots \\
x_{\beta_{n}}
\end{array}\right)=0 .
$$


As in (i), we forget $R_{n}$. Then $p_{i}$ and $f_{i}$ are elements of $\underset{k=0}{\oplus-1} C_{k}$. Using the monomial

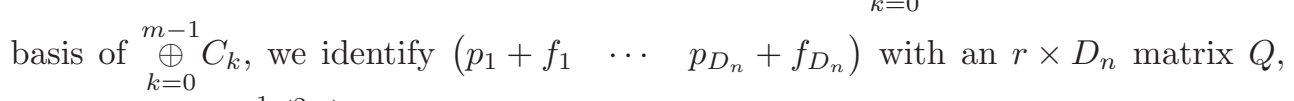
where $r=\sum_{k=0}^{m-1}\left(\begin{array}{c}2 m \\ k\end{array}\right)$ and an element of $Q$ is in $\mathbb{Z}_{2}$. Thus we obtain

$$
Q P\left(\begin{array}{c}
x_{1} \\
\vdots \\
x_{\beta_{n}}
\end{array}\right)=0 .
$$

The following result is proved in [16, Lemma 1]: For a fixed $v \in \mathbb{N}$, we set $X=$ $\{1, \cdots, v\}$. We also fix $s$ and $t \in \mathbb{N}$. For each $s$-element subset $\Delta$ of $X$, we consider the sum of $k$-element subsets of $\Delta$, where $k$ runs over $0 \leqslant k \leqslant t$. In the same way as in $Q$, we construct a $\left(\sum_{k=0}^{t}\left(\begin{array}{l}v \\ k\end{array}\right)\right) \times\left(\begin{array}{l}v \\ s\end{array}\right)$ matrix $U$, whose elements are in $\mathbb{Z}_{2}$. Assume that the condition $t \leqslant s \leqslant v-t$ is satisfied. Then we have $\operatorname{rank} U=\left(\begin{array}{l}v \\ t\end{array}\right)$. As remarked in $\left[\mathbf{1 6}\right.$, line 5], the result certainly holds for any field, in particular, for $\mathbb{Z}_{2}$.

Now setting $v=n-1, s=m+1$, and $t=m-1$, we have $\operatorname{rank} Q=\left(\begin{array}{c}2 m \\ m-1\end{array}\right)=D_{n}$. Therefore, the columns of $Q$ are linearly independent. From (18), we have

$$
P\left(\begin{array}{c}
x_{1} \\
\vdots \\
x_{\beta_{n}}
\end{array}\right)=0 .
$$

Since $\operatorname{rank} P=\beta_{n}$ by (i), we have $x_{1}=\cdots=x_{\beta_{n}}=0$. This completes the proof of Lemma 10 (ii).

Proof of Proposition 8 for $q=m-2$. We define a linear subspace $Y_{m}$ of $\mathbb{Z}_{2}\left[R_{n}\right.$, $\left.V_{1}, \ldots, V_{n-1}\right]$ by

$$
Y_{m}=\left\{R_{n}^{m-k} V_{i_{1}} \cdots V_{i_{k}} \mid 1 \leqslant i_{1}<\cdots<i_{k} \leqslant n-1 \text { and } 0 \leqslant k \leqslant m-2\right\} .
$$

Let $Z_{m}$ be the linear subspace of $\mathbb{Z}_{2}\left[R_{n}, V_{1}, \ldots, V_{n-1}\right]$ spanned by polynomials in $\left\{F_{\xi} \mid \xi \in \Omega_{m+1}\right\}$, where $F_{\xi}$ is defined in Lemma 10 (ii).

Then we have

$$
\operatorname{Im}\left[\cup R_{n}^{2}: H^{m-2}\left(\bar{M}_{n} ; \mathbb{Z}_{2}\right) \rightarrow H^{m}\left(\bar{M}_{n} ; \mathbb{Z}_{2}\right)\right] \cong Y_{m} / Z_{m}
$$

Note that $\operatorname{dim} Y_{m}=\gamma_{n}$. On the other hand, Lemma 10 tells us that $\operatorname{dim} Z_{m}=\beta_{n}$. Hence $\lambda_{m-2}=\gamma_{n}-\beta_{n}$.

\section{Proof of Theorem 6}

(i) We claim the following assertion: Let $\pi: X \rightarrow \bar{X}$ be a regular 2 -cover such that the groups $H_{*}(X ; \mathbb{Z})$ are torsion free. If $x \in H_{*}(\bar{X} ; \mathbb{Z})$ is a torsion element, then we have $2 x=0$.

The assertion is well-known. (See, for example, the arguments in [2, 9.3.2].) In fact, if $\operatorname{tr}^{*}: H_{*}(\bar{X} ; \mathbb{Z}) \rightarrow H_{*}(X ; \mathbb{Z})$ is the transfer homomorphism, then $\pi_{*} \circ \operatorname{tr}^{*}=2$.

Since the groups $H_{*}(X ; \mathbb{Z})$ are torsion free, we have $\operatorname{tr}^{*}(x)=0$. Hence $2 x=0$. 
Now if we apply the above assertion to the regular 2-cover

$$
\pi: M_{n} \times S^{1} \rightarrow E_{n}
$$

then Theorem 6 (i) follows.

(ii) We have

$$
\begin{aligned}
H_{*}\left(E_{n} ; \mathbb{Q}\right) & \cong H_{*}\left(M_{n} \times S^{1} ; \mathbb{Q}\right)^{\tau_{*}} \\
& \cong H_{*}\left(M_{n} ; \mathbb{Q}\right)^{\tau_{*}} \otimes H_{*}\left(S^{1} ; \mathbb{Q}\right) \\
& \cong H_{*}\left(\bar{M}_{n} ; \mathbb{Q}\right) \otimes H_{*}\left(S^{1} ; \mathbb{Q}\right) .
\end{aligned}
$$

It is known in $\left[\mathbf{7}\right.$, Theorem C] (see also [6]) that $P S_{\mathbb{Q}}\left(\bar{M}_{n}\right)=\phi_{n}$. Hence (19) is as given in Theorem 6 (ii).

(iii) We claim that

$$
P S_{\mathbb{Z}_{2}}\left(E_{n}\right)=\sum_{q=0}^{m-2}\left(\begin{array}{l}
n \\
q
\end{array}\right) t^{q}+\left(\left(\begin{array}{c}
n \\
m-1
\end{array}\right)+\beta_{n}\right)\left(t^{m-1}+t^{m}\right)+\sum_{q=m+1}^{n-2}\left(\begin{array}{c}
n \\
q+2
\end{array}\right) t^{q} .
$$

To prove this, we consider the Gysin sequence of the bundle (9):

$$
\begin{aligned}
\cdots \longrightarrow H^{q-2}\left(\bar{M}_{n} ; \mathbb{Z}_{2}\right) \stackrel{\cup R_{n}^{2}}{\longrightarrow} H^{q}\left(\bar{M}_{n} ; \mathbb{Z}_{2}\right) \longrightarrow H^{q}\left(E_{n} ; \mathbb{Z}_{2}\right) \\
\longrightarrow H^{q-1}\left(\bar{M}_{n} ; \mathbb{Z}_{2}\right) \stackrel{\cup R_{n}^{2}}{\longrightarrow} \cdots
\end{aligned}
$$

To check that the homomorphism is certainly $\cup R_{n}^{2}$, note that (9) is the sphere bundle associated to the vector bundle $M_{n} \times_{\tau} \mathbb{R}^{2} \cong \xi \oplus \xi$. Using Theorem 7 (ii), we have $w_{2}(\xi \oplus \xi)=w_{1}(\xi)^{2}=R_{n}^{2}$. Hence the homomorphism is $\cup R_{n}^{2}$.

From exactness, we have

$$
\operatorname{dim} H^{q}\left(E_{n} ; \mathbb{Z}_{2}\right)=\operatorname{dim} H^{q-1}\left(\bar{M}_{n} ; \mathbb{Z}_{2}\right)+\operatorname{dim} H^{q}\left(\bar{M}_{n} ; \mathbb{Z}_{2}\right)-\lambda_{q-2}-\lambda_{q-1} .
$$

Using (11) and Proposition 8, we obtain (20).

Now the universal coefficient theorem tells us that

$$
\Gamma\left(E_{n}\right)=\frac{P S_{\mathbb{Z}_{2}}\left(E_{n}\right)-P S_{\mathbb{Q}}\left(E_{n}\right)}{1+t} .
$$

Hence Theorem 6 (iii) follows.

\section{Proof of Theorem 5}

Consider the Wang sequence of the bundle (9) (see, for example, [12, Lemma 8.4]):

$$
\begin{gathered}
\cdots \longrightarrow H_{m-1}\left(M_{n} ; \mathbb{Z}\right) \stackrel{1-\tau_{*}}{\longrightarrow} H_{m-1}\left(M_{n} ; \mathbb{Z}\right) \longrightarrow H_{m-1}\left(E_{n} ; \mathbb{Z}\right) \\
\longrightarrow H_{m-2}\left(M_{n} ; \mathbb{Z}\right) \stackrel{1-\tau_{*}}{\longrightarrow} H_{m-2}\left(M_{n} ; \mathbb{Z}\right) \longrightarrow
\end{gathered}
$$

(i) The case where $m$ is even. We claim that the homomorphism

$$
1-\tau_{*}: H_{m-2}\left(M_{n} ; \mathbb{Z}\right) \rightarrow H_{m-2}\left(M_{n} ; \mathbb{Z}\right)
$$

is zero. In fact, the following commutative diagram tells us that it will suffice to prove 
the assertion for $\left(S^{1}\right)^{n-1}$ and the result is clear.

$$
\begin{array}{ccc}
H_{m-2}\left(M_{n} ; \mathbb{Z}\right) & \stackrel{1-\tau_{*}}{\longrightarrow} & H_{m-2}\left(M_{n} ; \mathbb{Z}\right) \\
i_{*} \downarrow \cong & \cong \downarrow i_{*} \\
H_{m-2}\left(\left(S^{1}\right)^{n-1} ; \mathbb{Z}\right) & \underset{1-\tau_{*}}{\longrightarrow} H_{m-2}\left(\left(S^{1}\right)^{n-1} ; \mathbb{Z}\right) .
\end{array}
$$

By (1), we have

$$
H_{m-1}\left(M_{n} ; \mathbb{Z}\right)=\underset{2 D_{n}}{\oplus} \mathbb{Z}
$$

Since $m$ is even, Theorem 6 tells us that

$$
H_{m-1}\left(E_{n} ; \mathbb{Z}\right)=\underset{\left(\begin{array}{c}
n-1 \\
m-2
\end{array}\right)+D_{n}}{\oplus} \mathbb{Z} \oplus \underset{\beta_{n}}{\oplus} \mathbb{Z}_{2}
$$

From exactness, an elementary divisor of the homomorphism (8) is 0,1 , or 2 . The numbers of the elementary divisors 2 and 0 are $\beta_{n}$ and

$$
\left(\begin{array}{c}
n-1 \\
m-2
\end{array}\right)+D_{n}-\left(\begin{array}{c}
n-1 \\
m-2
\end{array}\right)=D_{n}
$$

respectively. Hence the number of the elementary divisor 1 is

$$
2 D_{n}-\left(\beta_{n}+D_{n}\right)=\alpha_{n}
$$

(ii) The case where $m$ is odd. The homomorphism $(21)$ satisfies that $\left(1-\tau_{*}\right)(x)=$ $2 x$ for all $x \in H_{m-2}\left(M_{n} ; \mathbb{Z}\right)$. Using this, we can determine the elementary divisors of (8) in the same way as in (i).

\section{Proofs of Theorem 2 and Corollary 3}

Lemma 11. For $x, y$, and $z \in \mathbb{N} \cup\{0\}$, we define a square matrix of size $2 x+y+z$ by

$$
F(x, y, z)=\oplus_{x}\left(\begin{array}{ll}
0 & 1 \\
1 & 0
\end{array}\right) \oplus \underset{y}{\oplus}(1) \oplus \underset{z}{\oplus}(-1) .
$$

Then the following results hold.

(i) Let $P$ be an integral square matrix such that $P^{2}=I$. Then there exist $x, y$, and $z \in \mathbb{N} \cup\{0\}$ such that $P$ and $F(x, y, z)$ are integrally similar, that is, there exists $S \in G L(n, \mathbb{Z})$ such that

$$
S^{-1} P S=F(x, y, z) .
$$

(ii) If $(x, y, z) \neq(p, q, r)$, then $F(x, y, z)$ and $F(p, q, r)$ are not integrally similar.

Proof. (i) Using [10, p. 9, Example] (see, in particular, $B_{m}$ in $[\mathbf{1 0}$, p. 10]), $P$ is 
integrally similar to

$$
Q:=\left(\begin{array}{cc}
I_{r} & \left(\begin{array}{cc}
I_{k} & 0 \\
0 & 0
\end{array}\right) \\
0 & -I_{s}
\end{array}\right)
$$

for some $k$ with $0 \leqslant k \leqslant \min (r, s)$. Since $Q$ is integrally similar to

$$
\underset{k}{\oplus}\left(\begin{array}{ll}
0 & 1 \\
1 & 0
\end{array}\right) \underset{r-k}{\oplus}(1) \oplus \underset{s-k}{\oplus}(-1),
$$

(i) follows.

(ii) If $F(x, y, z)$ and $F(p, q, r)$ are integrally similar, then so are $I-F(x, y, z)$ and $I-F(p, q, r)$. An elementary divisor of $I-F(x, y, z)$ is 0 , 1 , or 2 such that their numbers are $x+y, x$, and $z$ respectively, and a similar result holds for $I-F(p, q, r)$. The assumption $(x, y, z) \neq(p, q, r)$ implies that $(x+y, x, z) \neq(p+q, p, r)$. Hence (ii) follows.

Proof of Theorem 2. By Lemma 11 (i), $A$ is integrally similar to $F(x, y, z)$ for some $x, y$, and $z \in \mathbb{N} \cup\{0\}$. Using Theorem 5, we compare the numbers of the elementary divisors 0,1 , and 2 of $I-A$ and $I-F(x, y, z)$. Consequently, we have $x=\alpha_{n}$ and $y=z=\beta_{n}$.

Proof of Corollary 3. The right-hand side of (6) is $F(d, 0,0)$ and that of (7) is $F\left(\alpha_{n}, \beta_{n}, \beta_{n}\right)$. Since $\beta_{n} \neq 0$ for $n \geqslant 7$, Lemma 11 (ii) tells us that the right-hand sides of (6) and (7) are not integrally similar.

\section{References}

[1] S. Bell, P. Jones, and J. Siemons, On modular homology in the Boolean algebra. II, J. Algebra 199 (1998), 556-580.

[2] A. Degtyarev, I. Itenberg, and V. Kharlamov, Real Enriques Surfaces, Lecture Notes in Mathematics 1746, Springer-Verlag, Berlin, 2000.

[3] M. Farber, J.-C. Hausmann, and D. Schütz, On the conjecture of Kevin Walker, J. Topol. Anal. 1 (2009), 65-86.

[4] M. Farber and D. Schütz, Homology of planar polygon spaces, Geom. Dedicata 125 (2007), 75-92.

[5] J.-C. Hausmann, Sur la topologie des bras articulés, Lecture Notes in Mathematics 1474, Springer-Verlag, Berlin, 1989, pp. 146-159.

[6] J.-C. Hausmann and A. Knutson, The cohomology ring of polygon spaces, Ann. Inst. Fourier (Grenoble) 48 (1998), 281-321.

[7] Y. Kamiyama, Topology of equilateral polygon linkages in the Euclidean plane modulo isometry group, Osaka J. Math. 36 (1999), 731-745.

[8] Y. Kamiyama and M. Tezuka, Topology and geometry of equilateral polygon linkages in the Euclidean plane, Quart. J. Math. 50 (1999), 463-470.

[9] M. Kapovich and J. Millson, On the moduli space of polygons in the Euclidean plane, J. Differential Geom. 42 (1995), 430-464. 
[10] T.J. Laffey, Lectures on integer matrices, unpublished lecture notes (1997). Available at http://hermite.cii.fc.ul.pt/meetings/im_1997/ lectures.pdf.

[11] R.J. Milgram and J.C. Trinkle, The geometry of configuration spaces for closed chains in two and three dimensions, Homology Homotopy Appl. 6 (2004), 237267.

[12] J. Milnor, Singular points of complex hypersurfaces, Ann. of Math. Stud., vol. 61. Princeton Univ. Press, Princeton, NJ, 1968.

[13] D. Schütz, The isomorphism problem for planar polygon spaces, J. Topol. 3 (2010), 713-742.

[14] N.J.A. Sloane, The On-Line Encyclopedia of Integer Sequences. Available at http://oeis.org.

[15] K. Walker, Configuration spaces of linkages, Undergraduate thesis, Princeton (1985).

[16] R. Wilson, A diagonal form for the incidence matrices oft-subsets vs. $k$-subsets, European J. Combin. 11 (1990), 609-615.

Yasuhiko Kamiyama kamiyama@sci.u-ryukyu.ac.jp

Department of Mathematics, University of the Ryukyus, Nishihara-Cho, Okinawa 903-0213, Japan 